\title{
Evaluation of Functional Parameters of Vehicle Simulators
}

\author{
Grzegorz Gudzbeler, Andrzej Urban, and Krzysztof Lojek
}

\begin{abstract}
The authors presented in the paper method of evaluation of operational performance of vehicle simulators. Numbers of simulation solutions are created in conjunction with various requirements for the quality of simulation, which make the audience difficult to define requirements and the authors do not always reach desired effects due to budget constraints. The paper presents the idea for the evaluations of the functional parameters of vehicle simulators. It contains essential information both for creators and consumers of such solutions. Authors present also a comparative study aimed at answering the question about quality of projection systems designed for vehicle simulators. They decided to make an attempt to give the preliminary answer to a question on which studied projection systems causes lesser degree of symptoms of simulator sickness during training. For the purposes of examinations two test platforms were prepared. One was equipped with a screen with a cylindrical projection system, the second with "on screen" projection system. This paper presents the results of comparative tests carried in consortium by Police Academy in Szczytno, Poland, as a part of scientific project "Building simulator of driving privileged vehicles in typical and extreme situations".
\end{abstract}

Index Terms-Simulation, evaluation, vehicles, training.

\section{INTRODUCTION}

Modern technologies allow for application of the modern methods of support for the process of teaching in the form of trainers using a computer simulation. Training simulation has been becoming a very important element in the process of teaching. Schemes of this type have varying degree of complexity. Starting from the solutions designed for handling of equipment exercises, through systems that enable the exercise of basic skills such as virtual shooting ranges, to complex simulation systems using distributed simulation architecture, enabling multiple users to simultaneously exercise at different levels of action. The task of software to generate a virtual environment is to replicate the realities of the real world in a virtual environment so that the person doing the exercise has been subjected to stimuli with a high degree of realism. What makes some games more engaging than others? According to the Lazzaro model [1] there are four keys indicatingwhy people enjoy playing certain games, each key representing a different aspect of fun: "hard" fun, "easy" fun, "people" fun, and "serious" fun. Games that incorporate meaningful challenges, strategies, and puzzles offer hard fun - a compelling balance of game difficulty with player skill through levels, player progress, or player controlled choices. For many people, the psychological rewards of achievement and triumph experienced after

Manuscript received June 15, 2013; revised September 10, 2013

The authors are with the Policee Academy in Szczytno, Poland (tel.: +4889621 5181; e-mail: g.gudzbeler@wspol.edu.pl, a.urban@wspol.edu.pl, xterra65@wp.pl). overcoming obstacles elicits positive emotions [2]. Easy fun is associated with games that allow for creative exploration and imaginative role-play. Easy fun is often a result of a game being immersive, because immersion appeals to a player's sense of curiosity, surprise, awe and wonder and keeps them engaged. Also, many games are appealing because they stimulate social bonding and interpersonal interaction (or at least the illusion of interaction), and therefore engender users' engagement via people fun. Serious fun is played with a purpose. Thus, "serious games" are designed to create or express value in play. Unfortunately, in the case of the training routine using simulation system based on complete scenarios can lead to disappearance of all these elements that cause the interest and commitment to practicing. The functioning of such systems with permanently defined scenarios makes trainee to learn the script, and each subsequent exercise has less and less educational value. Person doing the exercise stops responding appropriately to stimuli coming from the system, giving an appropriate response. Assessment of the quality of the trainee to prepare the appropriate response to real situations can thus be biased.

The scenario is normally understood as a whole, the events and their outcomes defined in the appropriate places in a virtual environment place on the correct position on the timeline. This approach to simulation results that we know its course in the elements controlled by AI from start to the end. This process is no longer a mystery to the practitioner after the first simulation. Therefore, the multiple repetition of exercises on simulators with a small number of scenarios prevents the achievement of the objectives of training (it becomes useless). The solution is to treat the scenario as an exercise of course dependent on the behavior of the practitioner with the individual events added before and after a course of exercises by the instructor. Events added by the instructor, called injects, should have the independence and completeness, so that they can be added at any time and repeated during the exercise. Other elements in the simulator which determine the functional properties are the moving platform, integrated visualization environment and sounds. Their design and parameters should be selected so that the person using system, regardless of its destination (for purposes of training or research) should in all driving conditions have feelings as close to those that would in the real world as possible. Of course for system evaluation purposes, reactions of the same person should be compared, because different people in the same circumstances may have different behavior. To do so, validation studies should rely on the measurement of certain quantities related to the moving platform, vehicle motion parameters and a broad range of reactions of the driver (the impact on vehicle traffic control authorities such as the steering, clutch pedal, brake and gas, their mental or physical conditions, eye tracking, etc.). Of course the ideal would be if corresponding values obtained 
for two systems were the same (absolute validation). In practice, however, it is impossible to achieve. Therefore, the current prevailing view is that the quality of the simulator is sufficient (validation relative), if the direction of changes are the same and values are similar or identical in both real and virtual systems. Authors of some works [3] point to the fact that, despite the similar behavior of drivers in the simulator and on the road, some imperfections in the projected image can cause that not all signals from the environment can be perceived by trainee, and lower resolution cause that many details may not be seen and lighting conditions can be different from the real world. Simulator validation studies should include not only a comparison of such quantities as the speed and trajectory of the vehicle, but also aspects of risk including driver's ability to adapt to a particular dangerous situation on the road.[4] An important factor influencing the reactions of the person using the driving simulator is the mutual coupling between man and machine in short called HMI (Human-Machine-Interfaces).

\section{EVALUATION OF FUNCTIONAL PARAMETERS}

The objective of the HMI evaluation in the simulator is to investigate behavior of the driver according to the following three components [5]:

1) The parameters characterizing the driver (age, experience, gender, fatigue, effects of drinking alcohol, taking drugs, mental or physical conditions, etc.)

2) The parameters characterizing the vehicle (dynamic properties that determine its response to the change in the position of the controls, the characteristics of their charges, etc.)

3) Parameters characterizing the traffic, current traffic situation and the external environment (graphic representation of the surrounding properties including: all signs on the road, control lights at road junctions and pedestrian crossings, buildings and other immovable objects, surrounding area, moving or standing vehicles of various types, different weather conditions such as rain, snow, fog, different times of day such as sunrise, midday, dusk, night).

Simulator, the performance properties are assessed should have the same features as the actual vehicle (seats, dashboard, pedals, steering wheel, radio, CD, navigation, storage space), including devices to assist it while driving (ABS, ECS, TCS, $4 \mathrm{WS}$, power steering, navigation) and warn the driver of the danger (parking sensors, liquid level indicators, indicators of a malfunctioning engine). To fully assess the impact of construction on the nature of the simulator coupling between man and machine, which will later decide about its usefulness in the process of training or research, advanced mobile simulator should be used for testing. In order to compare the values obtained from tests on the simulator to test in real conditions, it is needed to use a lot of instruments which will be able to measure the following quantities:

1) Coordinates of the location of the center point of the vehicle and the traffic parameters (speed, acceleration, direction, velocity and angular acceleration),

2) The location and movement of other vehicles that are simulated in its environment,
3) Position of elements controlled by the driver (steering wheel, pedal brake, clutch and gas, buttons), and eventually force or torque applied to them,

4) The psychophysical parameters of the driver (tracking eye position and look direction, monitoring of ECG and EEG, respiratory rate, pulse measurement, the impedance of the skin, electromyography,

5) The driver's acceleration along three axes.

6) Observation of the behavior of the driver by a camera with video recording.

7) The archiving of the situation on the road.

8) The current record images on the screen.

Measurement data should be available in real time or later after performed tests for analysis. In addition to the above, the essential element of the evaluation can also be a specially prepared questionnaire filled in by the driver immediately after the test or sometime after. It can provide additional information, which is not able to detect by a measuring system, such as any finding of simulator sickness. It has a vital importance in assessing the operational properties of a driving simulator. Simulator's sickness is a term used to describe a variety of adverse reactions of persons under training on simulators of moving targets - aircrafts, marine and ground vehicles [1]. Due to the increasingly widespread use of simulators for training operators of various moving objects, literature is very rich, but focused mainly on pilot training aircraft. In the analyzed literature, there is no conclusive resolution of the causes and prevention of simulator sickness. This illustrates the complexity of the reasons causing it, which is the subject of intensive research. For purposes of this study term simulator sickness is used, regardless of simulator platform. The most general statement is that "simulator sickness has many symptoms and largely depends on the characteristics between individuals".

The experimental studies show that the simpler exercises of tasks described below and maneuvers for which the angular velocity of the deflection does not exceed $8^{\circ} / \mathrm{s}$ and lateral acceleration of $3.3 \mathrm{~m} / \mathrm{s}^{2}$ - a much less expensive stationary driving simulator can be used. These are the most frequent activities while driving, such as:

1) Ride along the same lane at a constant speed.

2) A smooth acceleration and braking,

3) To perform soft turns.

4) Tasks associated with a gradual change in lane and carrying out activities related to the soft turning of the steering wheel, assuming that a high quality system to generate resistance in the steering system is provided.

The use of the simulator with the moving stand is certainly justified in the case of more complex tasks and maneuvers, such as:

1) The wheel turns or abrupt maneuvers with rapid acceleration or deceleration, in which we are dealing with significant acceleration.

2) Driving in a circle.

3) Violent maneuvers in an urban environment especially performed at low speeds.

4) All maneuvers with frequent changes of direction.

5) Driving over uneven, unpaved roads, potholes, etc.

6) Emergency maneuvers, while avoiding collisions, pursuits, etc. 
Evaluation of the HMI (coupling between man and machine operator or a simulator) consists of two stages. The first one is to determine certain common tasks that are to carry out by the driver, such as:

1) Navigating along the route (see route along with any additional instructions).

2) Driving along a road on the screen generated by the computer graphics.

3) Maintaining a constant speed (acceleration and braking).

4) Responding to the specific situation on the road.

5) Detecting and bypassing obstacles.

6) Other than those mentioned in line with the assumed scenario.

In addition to the system can be implemented noise is for example the influence of variables associated with side forces of wind. During the first stage consisting of the above described tasks the following parameters associated with the movement of the car and driver activity are typically recorded:

1) The current position of the controls (steering wheel, brake pedal, gas pedal), through which the driver controls the movement of the car,

2) The longitudinal and lateral acceleration.

3) The angular velocity of the car turnover and its direction.

4) The speed of movement.

5) The current position in the adopted coordinate system fixed.

In the second stage of the evaluation of the quality man-machine coupling, measurement of driver activity during the test is required. Special equipment is used which allow measurement of the rate of reaction, and deviations from established patterns and also viewing the behavior of the driver and observation of their reactions (the camera in the cockpit), etc.

Number of participants in the experiment is not easy to determine and depends on many aspects and especially on the purpose of the evaluation. Generally, the more participants, the research is more accurate, but at the same time many aspects, like budget, are limiting their number. In preliminary tests, it seems reasonable to involve a homogeneous group of participants of similar age and experience, it should be 16-20 persons according to the ISO 26022 "Lane Change Test", which recommends testing for at least 16 people. In other ISO 16673 standards, this number is 10. Taking this into account, it means that examination of 16 participants is sufficient to correctly assess the HMI, yet the acceptable minimum is 10 people. Taking into account the fact that typically about $8-10 \%$ of people suffer from a disease simulator, it is reasonable to start with about 20 people. Objective evaluation indicators can be implemented either in the main computer or in special module which can be plug in via an interface to the simulator during the test.

Now a few examples of validation of driving simulator will be discussed. In the first one performed at the University of Leeds [6], [7] a static simulator was used, in which 100 drivers were tested: 50 men and 50 women in different age groups. Most of the respondents did not exceed thirty years of age, and had a driving license for less than 5 years. They came mainly from the university; they had not experiences in "driving" a car in a virtual environment and were not advanced in computer games. The study was carried out under real conditions on two-lane road, six kilometers long a fragment located outside the city, consisting of straight lines and a few curves. On the way there was a medium and low traffic volume (average of 6800 vehicles per day), with approximately $20 \%$ were heavy goods vehicles and $80 \%$ of cars and vans. In a virtual environment, some efforts were made to faithfully reproduce the real conditions up. Vehicle speed in the 21 measuring points of road was checked both on real road and in the simulator, and compared their values with each other by highlighting cases of driving on the straight sections of road and the bends in the absence of other vehicles on the road and in their presence. It turned out that in the straight sections of the simulator, driver went faster on average, about $9 \mathrm{~km} / \mathrm{h}$ with traffic and about $11 \mathrm{~km} / \mathrm{h}$ without, and drove slower on curves by less than $5 \mathrm{~km} / \mathrm{h}$. Impact of road traffic in this case was small. Since the average speed was about $65 \mathrm{~km} / \mathrm{h}$, the speed differences were within the range of $6-19 \%$, and standard deviations amounted to $2-5 \%$ of the average.

Another example of an advanced driving simulator validation studies were discussed in [8] involving a comparison of changes in average speed during three maneuvers: braking before the "stop" sign and turn to the right and left with an angle of $150^{\circ}$ while driving in the simulator and accordingly equipped car. Authors also compared the maneuvers on the standard road (the control site) and the "convex" cross painted in red stripes with a width of $60 \mathrm{~cm}$ and a height of $1 \mathrm{~cm}$ (the treatment site). Drive through lanes prior to crossroads or curve at about 30-50 m (treatment area) was clearly felt by the driver and act as a feedback signal. The distance between the belts has been progressively reduced as you approach a junction or bend. All maneuvers were performed on two-lane suburban road near Melbourne and the driving simulator installed at the Monash University Accident Research Centre (MUARC). It had a fully equipped cabin of Ford Falcon. The image had a resolution of $640 \times 480$ pixels and was displayed at a frequency of $30 \mathrm{~Hz}$. The range of visibility at the front was $180^{\circ}$ (three screens and projectors), back $60^{\circ}$ (four screens and a projector) and vertically $45^{\circ}$. The simulator had a sound generator to simulate the various sounds coming from the engine and from outside the car. Base simulator was mounted on a movable platform in order to simulate acceleration, braking, taking turns and overtaking. The study involved 24 drivers, 12 men and 12 women aged from 22 to 52 years old (average 29.8 years). All participants had completed their studies and each had a driving license and at least 3 years' experience in driving. No one had ever been in a car simulator or in a flight simulator. Before proceeding to the appropriate research participants were acquainted with the instructions and have completed a test drive that lasted about $11 \mathrm{~min}$. The experimental drive lasted from 40 to 50 minutes depending on the driver and the circumstances. For the experiments used a suitably equipped Holden Apollo with an engine capacity of 21 speed was measured by the speedometer, which was connected to the vehicle unit. Speed values in both cases were recorded at a frequency of $30 \mathrm{~Hz}$ and then converted to an average speed of $1 \mathrm{~m}$ distance traveled. The measurement started always at the same 
distance from the belt corresponding to approximately the same length, which are distributed stripes. Study was conducted to perform absolute and relative validation. Basis on that the following conclusions were made:

1) Studies both in real and virtual environment shown that in all cases the speed reduction takes place even before the strips.

2) The introduction of lanes before the intersection with a "stop", causes a marked reduction in average travel speed for both tests on the road and in the simulator.

3) The difference between the average speeds obtained on the roads with lanes and roads without lanes for the maneuver associated with the bend is greater when driving simulator then in real conditions. This may result from both the properties of the simulator and the experimental procedures used. In the absence of a signal coming from the inertia of motion, the driver may be less aware of changes in speed, which is associated with "worse" feeling of the car during braking,

4) The general accuracy of the resulting study is that the speed achieved when driving in the simulator was in most cases lesser than the velocity obtained in the same conditions and at the same maneuvers, but when driving in the real world.

The research discussed in [8] showed that a comparative analysis of medium-velocity profiles obtained during the same conditions and maneuvers in the real world and virtual (driving simulator) is an appropriate criterion for both absolute and relative validation of driving simulators. Similar studies carried out on two-lane road outside the city are discussed in [9].

A similar assessment of the operational properties of the simulator by comparing the reactions and behavior of drivers during real driving, while watching a short video showing the vehicles moving in traffic and when driving a stationary simulator equipped with a projection system with viewing angle of $90^{\circ}$ presents the work of [10]. Authors believe that the validation of simulation involving only a comparison of the speed and degree of adherence to the belt path is limited to a lower level of car control takes into account the mainly perceptual-motor characteristics associated with driving a car and is a necessary condition but not sufficient to fully assess operational properties of a simulator. The authors propose to introduce a higher level of assessment of vehicle control associated with cognitive aspects. As in the case of comparing the size of the previously mentioned factors it seems important to compare the driver's awareness by observing the changes in behavior in specific situations in driving associated with increased observation of the environment. If an experienced driver can sense danger on the road, then occur a change in his way of tracking what is happening currently on the road. It is showed it in works of Crundall [11] and Underwood [12]. In studies they showed that more experienced drivers observe the traffic situation in the wider area and are characterized by shorter time intervals in which their point of view is fixed. Validation of the simulator that was used in the work consisted in the observation of behavior patterns of both experienced and novice drivers. Due to the large variety of dangerous situations and their variants, which may take place on the road, proper selection is very important. For each of them you can define multiple variables that will be used for risk assessment. Among them, select those items that are most relevant in the assessment of training of both drivers and evaluation of the simulator. A more detailed description of the research can be found in [10].

\section{IMPACT OF PROJECTION SYSTEMS FOR VEHICLE}

SIMULATORS ON SYMPTOMS OF SIMULATOR SICKNESS

Simulator's disease is a condition characterized by a number of symptoms in extreme conditions: nausea, vomiting, pallor, and increased sweating. They occur in humans under conditions of exposure to virtual or real visual motion stimuli, associated or not with kinetic stimuli. Those incentives are not physiological for a human and they are not adapted with humans. This definition is a broad concept encompassing: simulator, motion, air, maritime, automotive and space sickness, etc. The negative impact of the virtual environment of simulator to humans is therefore undesirable. For the first time the phenomenon was studied by Miller and Goodson (1958, 1960), who had symptoms that occur as a result of training in a simulator called motion sickness, because of the similarity of most of the symptoms of this disease to balance disorders. According to factors that affect the human body we can divide simulators on those where only exclusively kinetic incentives are used (Coriolis sample), with kinetic and visual incentives (simulators with visual stimuli on mobile platforms) and with only visual incentives (stationary simulators with visual stimulation). Due to this a simulator sickness name is more associated with the device on which symptoms can be occurred than with the phenomenon itself. Therefore, if the negative impact of the virtual environment simulator, regardless of the nature of the stimulus, we most often use name simulator sickness in relation to the set of symptoms occurring as a result of training on the simulator. Simulator disease is characterized by a rich and diverse symptomatology depending on the degree of its advancement. It often starts as stomach discomfort, bodily warmth, headache, dizziness and/or drowsiness, then proceeds to stomach distress, then nausea and vomiting. There are increasing nausea, often accompanied by symptoms of hypersensitivity to unpleasant taste and olfactory sensations, loss of appetite, headache, anxiety, adding to the ataxia and spatial disorientation. Due to the intensive tracking a virtual image at all times during examination the feeling of fatigue is common with blurred vision. In extreme cases the disease may be associated with violent vomiting, fatigue, apathy, and drowsiness and reduced mental capacity for concentration and muscle activity. Different configurations of symptoms in individual participants depend on the sensitivity of individual acting stimulus, the nature of the stimulus, the level and duration of action. Currently there is no conclusive statement about causes and prevention for simulator sickness. In the simulation of mobile objects, there are three main methods of visualization: on a helmet, on windows of a vehicle and on an 
external display.

\section{Test Platforms SPECIFICATION}

For the purposes of examinations two test platforms were prepared. One equipped with a screen with a cylindrical projection system, the second with "on screen" projection system.

Simulator with cylindrical projection system.

- Cabin - of intercity bus Autosan A1012T Leader

- Screen (cylindrical: with radius $\mathrm{R}=4.1 \mathrm{~m}$ and a height $\mathrm{h}=$ $3.75 \mathrm{~m}$, angles of sight from a point of view of the driver: angle width: vfov $=180 \mathrm{deg}$, angle height hfov $=50 \mathrm{deg}$ )

- Projection system (four projectors Projectiondesign F22 $\mathrm{SX}+, 1400$ x 1050 resolution, brightness - 2100 ANSI lumens, contrast ratio: 2500:1, type of matrix: DLP)

That made the projection system provided an angular resolution in front of the driver's sight - 2.9 arc minute / pixel.

Simulator with on screen projection system.

- Cabin-Mercedes Acros truck.

- Screen - "on screen" - stuck projection foil to all front and side windows allowing view using the rear projection type, rear windows were completely blacked out.

- Projection system:

1) 3 ultra-short throw projector Mitsubishi WD380U-EST serving front and left window (brightness: 2800 ANSI lumens, resolution: $1280 \mathrm{x}$ 800, contrast ratio: 3000:1, type of matrix: DLP),

2) 1 projector Panasonic PT-LB1E displays the image on the right window (brightness: 2200 ANSI lumens, contrast ratio: 500: 1 , resolution: 1024 x 768, type of matrix: LCD).

That made the projection system provided an angular resolution in front of the driver's sight - 2.1 arc minute / pixel.

\section{STUDY RESUltS}

The study was performed on 15 individuals who have not previously practiced on simulators. Number of participants in the experiment is not easy to determine and depends on many aspects and especially on the purpose of the evaluation. Generally, the more participants, the research is more accurate. In preliminary tests, it seems reasonable to involve a homogeneous group of participants of similar age and experience. In ISO 16673 standards, sufficient number of participants is 10. Taking this into account, it means that examination of 15 participants is sufficient; the acceptable minimum is 10 people.

Performed on these participants, a preliminary study has not identified diseases of their eye. Research on simulators with "on screen" and the cylinder projection system was performed at an interval of 10 days.

\section{A. Results of Research Conducted on the Simulator with Cylinder Projection System}

\section{1) The study carried out before training}

Interview: The interview with all participants indicated no disturbance, which could have an impact on training on the simulator. 3 people have symptoms of asthenopia negative (age-related abnormal accommodation, causing problems with reading without correction glasses).

Concerned ophthalmological examination:

1) The study of eye diseases. Refraction survey using computer autorefractometer in 11 participants showed a visual impairment that does not exceed $+/-1.5 \mathrm{D}$, the refractive state, which usually does not require a spectacle correction. 2 participants the defect was $-2.0 \mathrm{D}$, with a $-3.75 /-3.5 \mathrm{D}$, and a $-5.0 /-5.5 \mathrm{D}$. People with these defects are not excluded from training because according to the rules they may have a driving license.

2) The study of visual acuity. The visual acuity of the right and left eye in 10 participants ranged from $0.8-1.0$. In 5 participants it was within the limits $0.5-0.6$. So in any of the subjects, there was no reduction in visual acuity, which disqualifies them from driving.

3) Examination of the tear film with non-invasive test with a disruption of the tear film (NIBUT)) and the stability of the tear lipid layer films checked with Tearscope camera. NIBUT study showed normal values in all 15 participants (> $10 \mathrm{sec}$.). Examination of the lipid layer showed no abnormally thin in 13 participants (values A-C). In 2 of participants thickness of the lipid layer was thinned (E)

4) Examination of the binocular vision - Worth test. The study showed normal binocular vision in all participants.

5) The study of stereoscopic view - "Fly" test. Very good stereoscopy (Grade 8-9) occurred in 14 participants. A small reduction in stereoscopic occurred in 1 patient (grade 5).

6) The study of eyes setting - "cover test". In this study, there were no stability problems at the position of both eyes during their alternating covering (no small-angle strabismus and latent strabismus).

\section{2) The study carried out after training}

Interview: 12 persons after a training session on the simulator did not provide any information about visual disturbances. Three people gave out information about small disturbances in the form of "a strange image", "light disturbances when turning" and "strange impressions associated with non-motion simulator". These symptoms can be classified as a first degree of simulator sicknes in Chilow classification.

Concerned ophthalmological examination:

1) The study of visual acuity. The study showed no difference in visual acuity compared with state before training on the simulator. Small differences of 0.1 are within the error limits of the method.

2) Examination of the tear film with non-invasive test with a disruption of the tear film (NIBUT)) and the stability of the tear lipid layer films checked with Tearscope camera. NIBUT test showed no prolongation of the tear film break in all the participants. The study of the lipid layer showed no changes in its thickness in 14 participants. In 1 person was a small thinning of the layer thickness of 1 degree, but it was located within the normal range.

3) Examination of the binocular vision - Worth test. The study showed no changes in binocular vision in all participants after training. 
4) The study of stereoscopic view - "Fly" test. After training on the simulator, there was no reduction in stereoscopy in all subjects.

5) The study of eyes setting - "cover test". In this study there was no change in the position of both eyes after a training session on the simulator.

\section{B. Results of Research Conducted on the Simulator with "on Screen" Projection System.}

\section{1) The study carried out before training}

A. Interview In an interview in all participants there were no abnormalities that could have an impact on training on the simulator. 3 people have symptoms of asthenopia (age-related abnormal accommodation, causing problems with reading without correction glasses):

1) The study eye diseases. Refraction survey using computer autorefractometer in 11 participants showed a visual impairment does not exceed $+/-1.5 \mathrm{D}$, the refractive state, which usually does not require a spectacle correction. 2 participants the defect was $-2.0 \mathrm{D}$, with a $-3.5 /-3.25 \mathrm{D}$, and a -5.25 / -5.25 D. People with these defects are not excluded from training because, according to the Polish rules may they have a driving license (category A and B) in accordance with the Minister of Health regulation from 15 April 2011.

2) The study of visual acuity. The visual acuity of the right and left eye in 10 participants ranged from 0.8-1.0. In 5 participants it was within the limits 0.4-0.7. So in any of the subjects, there was no reduction in visual acuity, which disqualifies them to drive motor vehicles (Polish driving license category A and B) in accordance with the Minister of Health regulation from 15 April 2011.

3) Examination of the tear film with non-invasive test with a disruption of the tear film (NIBUT)) and the stability of the tear lipid layer films checked with Tearscope camera. NIBUT test showed normal values in 15 participants (> $10 \mathrm{sec}$.). Examination of the lipid layer showed no abnormally thin in 11 participants (the AC). In 4 of them thickness of the lipid layer was thinned (DE value).

4) Examination of the binocular vision - Worth test. The study showed normal binocular vision in all participants.

5) The study of stereoscopic view - "Fly" test. Very good stereoscopy (Grade 8-9) occurred in 14 participants. A small reduction in stereoscopic occurred in 1 patient (grade 5).

6) The study of eyes setting - "cover test". In the study, there was no evidence of impaired the stability of the position of both eyes during their alternating covering (no small-angle strabismus and latent strabismus).

\section{2) The study carried out after training}

Interview: 10 persons did not provide any visual disturbances after a training session on the simulator with an "on screen" projection system. 5 people reported the disorder in the form of "breathing", "a strange image," light nausea ," twisted image "and dizziness. These symptoms can be classified as 1 degree in 4 participants, and in one case as a second stage of simulator sickness in Chilow classification.

Concerned ophthalmological examination:

1) The study of visual acuity. The study showed no difference in visual acuity compared with state before training on the simulator. Small differences of 0.1 are within the error limits of the method.

2) Examination of the tear film with non-invasive test with a disruption of the tear film (NIBUT)) and the stability of the tear lipid layer films checked with Tearscope camera. NIBUT test showed no prolongation of the tear film break in all the participants. The study of the lipid layer showed no changes in its thickness in 14 participants. In 1 person was a small thinning of the layer thickness of 1 degree, but it was located within the normal range.

3) Examination of the binocular vision - Worth test. The study showed no changes in binocular vision in all participants after training.

4) The study of stereoscopic view - "Fly" test. After training on the simulator, there was no reduction in stereoscopy in 14 participants. In one person were reduced stereoscopic ranges of 1 degree (from 5 to 4 ).

5) The study of eyes setting - "cover test". In this study there was no change in the position of both eyes after a training session on the simulator.

\section{CONCLUSION}

Conducting research described above can improve the testing process of the evaluation terrestrial operating properties constructed driving simulator. They allow the phase of its development faster to obtain the required characteristics of the HMI. Why simulator sickness was more common in the simulator with the "on screen" projection system than a cylinder? It seems that this is due to the proximity of the screen. There is an analogy to the occurrence of symptoms when watching movies in $3 \mathrm{D}$. Watching the three-dimensional films at the cinema rarely causes simulator sickness because the screen is far from the spectators. The introduction of 3D technology for television meant that the symptoms began to be felt much more often. It is estimated that it may occur in $10-20 \%$ of people watching 3D TV. They sit closer to the screen, so the probability of that feeling is greater.

The eye study showed that training on simulators with "on screen" and the cylinder projection systems does not cause changes in the organ of vision in participants with the form of deterioration in visual acuity of the tear film, the state of binocular vision, stereoscopic view and eyes settings. Subjectively experienced symptoms of simulator sickness occurred less frequently after a training session on the simulator with a cylindrical screen than on the "on screen". It seems that the better tolerance of training on the simulator with cylinder screen is due to a greater distance from the screen.

\section{REFERENCES}

[1] N. Lazzaro, "The four keys to fun: Designing emotional engagement and viral distribution without spamming your friends," in Proc. ACM SIGCHI 2009, Palo Alto, USA, 2009.

[2] P. Eckman, Emotions revealed: Recognizing faces and feelings to improve communication and emotional life, NY: Henry Holt and Company LLC, USA, 2007.

[3] G. J. Blaauw, "Driving experience and task demands in simulator and instrumented car: A validation study," Human Factors, vol. 24, no. 4 , pp. 473-486, 1982. 
[4] X. Yan, M. Radwan, E. Wang, and X. P. Chilakapati, "Validating a driving simulator using surrogate safety measures," Accident Analysis and Prevention, vol. 40, pp. 274-288, 2008.

[5] D. Weir, "Application of a driving simulator to the development of in-vehicle human-machine-interfaces," IATSS Research (International Association of Traffic and Safety Sciences), vol. 34, pp. 16-21, 2010.

[6] E. Blana and J. Golias, "Behavioural validation of fixed-Base driving simulator," in Proc. Driving Simulation Conference, Paris, France, pp. 227-240, September 7-8, 1999.

[7] Facilities. [Online].

Available: http://www.its.leeds.ac.uk/facilities/lads/index.html

[8] S. Godley, T. J. Triggs, and B. N. Fildes, "Driving simulator validation for speed research," Accident Analysis and Prevention, vol. 34, pp. 589-600, 2002

[9] F. Bella, "Driving simulator for speed research on two-Lane rural roads," Accident analysis and Prevention, vol. 40, pp. 1078-1087, 2008.

[10] G. Underwood, D. Crundall, and P. Chapman, "Driving simulator validation with hazard perception," Transportation Research Part F, vol. 14, pp. 435-446, 2011.

[11] D. Crundall and G. Underwood, "The effects of experience and processing demands on visual information acquisition in drivers," Ergonomics, vol. 41, pp. 448-458, 1998.

[12] G. Underwood, P. Chapman, N. Brocklerhurst, J. Underwood, and D. Crundall, "Visual attention while driving: Sequences of eye fixation made by experienced and novice drivers," Ergonomics, vol. 46, pp. 629-646, 2003

Grzegorz Gudzbeler is a $\mathrm{PhD}$, who is the director of Institute for Coordination of Research and External Fundsin Police Academy in Szczytno He received his bachelor degree in mathematics from University in Bialystok, master degree in informatics from University of Computer Sciences and Economics in Olsztyn, Poland and doctor's degree in National Defense
Academy in Warsaw. He is author of many publications in topics of computer modeling and simulations, cybercrime and technical support for managing major events and crisis situations. He was engaged in project "Preparing Polish Police for Euro 2012”.Now is contractor in projects „Building Prototype Simulator of Police Operations in Crisis Situations”, "Building simulator of driving privileged vehicles in typical and extreme situations", and was engaged in preparing Polish Police for Euro Cup 2012.

Andrzej Urban is a PhD. Eng., who is Professor in Police Academy in Szczytno, Poland. He received engineer degree on University of Science in Gliwice , Poland and doctor degree on National Defense Academy in Warsaw, Poland. He is author of many publications in topics of computer modeling and simulations, crime prevention through environmental design and technical support for managing major events and crisis situations. He is manager of projects „Building Prototype Simulator of Police Operations in Crisis Situations" and "Building simulator of driving privileged vehicles in typical and extreme situations".

Krzysztof Lojek is the head of Department of Social Safety at the Institute of Security and Public Order (Police Academy in Szczytno, Poland). Since 1992 lecturer of matters of security and public order. He received his doctor's degree of humanities in security in National Defense Academy in Warsaw. Author of many publications in topics of crime prevention, community policing and crime prevention through environmental design. He was engaged in project "Transportable Autonomous Patrol for Land Border Syrveillance System ("TALOS") founded by the Seventh Framework Programme for Research and Technological Development. Now is contractor in "Building simulator of driving privileged vehicles in typical and extreme situations". 